\title{
Strong electron density modulation with a low-power THz source for generating $\mathrm{THz}$ superradiant undulator radiation
}

\author{
Gang Zhao, Sheng Zhao, Senlin Huang, ${ }^{*}$ and Kexin Liu \\ State Key Laboratory of Nuclear Physics and Technology and Institute of Heavy Ion Physics, \\ School of Physics, Peking University, Beijing 100871, China
}

(Received 1 May 2019; published 10 June 2019)

\begin{abstract}
We propose a scheme to modulate a low-emittance electron beam at $\mathrm{THz}$ frequency using a low-power optical THz source (seed). In this scheme, a matching section introduces tunable angular dispersion and focusing to the electrons. It helps to slice the electrons into "energy layers" and largely cancels out the impact of uncorrelated energy spread on electron modulation. Analytical and numerical studies have been carried out to demonstrate the proposed scheme. It is found that a strong electron density modulation (bunching) over the frequency range from 1 to $6 \mathrm{THz}$ can be achieved when the peak power of $\mathrm{THz}$ seed is no more than $1 \mathrm{~kW}$. The bunched beam can be used to generate superradiant undulator radiation with the peak power of 10 s MW.
\end{abstract}

DOI: 10.1103/PhysRevAccelBeams.22.060701

\section{INTRODUCTION}

Terahertz $(\mathrm{THz})$ radiation has many scientific and technological applications, such as molecular spectroscopy, remote sensing, security screening, medical, and biological imaging [1-3]. Among the numerous THz radiation sources, the accelerator-based $\mathrm{THz}$ sources are relatively complex but can generate high peak power beyond megawatt (MW) level $[4,5]$, which is considerably attractive for its unique application in driving new nonlinear phenomena of materials [6]. Recently, there has been great interest in generating intense narrowband $\mathrm{THz}$ radiation from electron beams bunched at picosecond (ps) and sub-ps scale [7-11]. For such a purpose, several electron density modulation (also referred to as "bunching") methods have been proposed, based upon techniques of phase space exchange $[12,13]$, photocathode drive laser modulation $[10,14]$, and electron energy modulation [9,15-23], etc.

The electron energy modulation can be induced by a wakefield [17-19] or an optical laser copropagating with the electron beam in an undualtor $[9,15,16,20-23]$. This energy modulation will then be converted into density modulation by dispersive optics, e.g., a magnetic chicane. As the development of laser technologies, laser-based electron bunching has been implemented widely, in which case the energy modulation is at the scale of the laser

\footnotetext{
"huangsl@pku.edu.cn

Published by the American Physical Society under the terms of the Creative Commons Attribution 4.0 International license. Further distribution of this work must maintain attribution to the author(s) and the published article's title, journal citation, and DOI.
}

wavelength. However, typical lasers used for energy modulation have a wavelength below $1 \mu \mathrm{m}$, which is much shorter than that of $\mathrm{THz}$ radiation. To modulate the electron beam for $\mathrm{THz}$ radiation, two novel schemes have been proposed. One is based on the echo-enabled harmonic generation (EEHG) technique [20-22], which uses two lasers with different wavelengths for electron energy modulation. The other is based on energy spread modulation of electron temporal slices [9], which uses a laser pulse with periodic intensity. Both schemes are supposed to generate density modulation at $\mathrm{THz}$ frequency.

Inspired by a recent technique proposed to introduce strong microbunching into the storage ring beam for generating coherent EUV and x-ray radiation [24], we propose a scheme to produce strong electron density modulation at $\mathrm{THz}$ frequency using an external $\mathrm{THz}$ source directly. In this scheme, the electron modulator comprises a matching section, an energy modulator resonant at $\mathrm{THz}$ frequency, and a dispersion section. The matching section introduces tunable angular dispersion and focusing to the electrons. It slices the electrons into "energy layers" and largely cancels out the degradation of electron modulation due to uncorrelated (initial) energy spread. In this case, the modulation induced energy deviation dominates the density modulation process, therefore only slight electron energy modulation is needed. The required peak power of seed radiation for the energy modulator is no more than $1 \mathrm{~kW}$, which is well within the reach of the state-of-the-art optical THz sources [25-29].

The remaining part of this paper is organized as follows. In Sec. II, we will first develop a complete analytical model for this study. We then present the scheme in Sec. III, followed by the design of a hypothetical beam line according to the model. In Sec. IV, a simulation study is carried out to 
validate the analytical result. In Sec. V, THz undulator radiation from the bunched electron beam is analyzed. Finally, we will give a brief summary in Sec. VI.

\section{THEORETICAL BASIS}

Let us start with a generalized configuration for electron density modulation, which comprises a matching section, an energy modulator, and a dispersion section. We assume the reference particle orbit is deflected in the horizontal $(x-z)$ plane only, therefore the electron modulation can be described in a 4-dimensional phase space with the coordinates defined by $\left(x, x^{\prime}, l, \delta\right)$, where $x$ is the horizontal coordinate, $x^{\prime} \equiv d x / d z$ is the horizontal divergence, $l$ is the longitudinal coordinate within the electron bunch, and $\delta$ is the relative energy deviation with respect to the reference particle.

Referring to the notations adopted in the widely-used $6 \times 6$ beam transport matrix [30], the $4 \times 4$ transfer matrices for the matching section and the dispersion section can be formulated as

$$
R_{a}=\left(\begin{array}{cccc}
a_{11} & a_{12} & 0 & a_{16} \\
a_{21} & a_{22} & 0 & a_{26} \\
a_{51} & a_{52} & 1 & a_{56} \\
0 & 0 & 0 & 1
\end{array}\right)
$$

and

$$
R_{b}=\left(\begin{array}{cccc}
b_{11} & b_{12} & 0 & b_{16} \\
b_{21} & b_{22} & 0 & b_{26} \\
b_{51} & b_{52} & 1 & b_{56} \\
0 & 0 & 0 & 1
\end{array}\right)
$$

respectively. The modulator, comprised of a horizontally polarized undulator, behaves as a linear transport element when the induced electron energy modulation is not included. The corresponding transfer matrix has the form [31]

$$
R_{m}=\left(\begin{array}{cccc}
1 & m_{12} & 0 & 0 \\
0 & 1 & 0 & 0 \\
0 & 0 & 1 & m_{56} \\
0 & 0 & 0 & 1
\end{array}\right),
$$

with $m_{12}=L_{u}+3 A_{2} / 2-3 A_{1}^{2} / 2 L_{u}$ and $m_{56} \approx L_{u} / \gamma_{0}^{2}+$ $\left(1+1 / 2 \gamma_{0}^{2}\right)\left(A_{2}-A_{1}^{2} / L_{u}\right)$. The scaled field integral factors $A_{1}=\left(e / p_{0}\right) \int_{0}^{L_{u}} d z \int_{0}^{z} B(\tau) d \tau$ and $A_{2}=\left(e / p_{0}\right)^{2} \int_{0}^{L_{u}} d z$ $\left[\int_{0}^{z} B(\tau) d \tau\right]^{2}$, in which $e$ is the electron charge, $p_{0}$ is the reference particle's momentum, and $B(z)$ is the on-axis undulator field. When the electron beam is highly relativistic and the undulator is short and has a symmetric field profile, $m_{12} \approx L_{u}$ and $m_{56} \approx 0$. Under such a condition, the electron energy modulation due to the interaction with a seed radiation in the undulator is equivalent to a timedependent energy kick exerted at the undulator exit. Therefore, a change to $\delta$ by an amount of

$$
\Delta \delta=\frac{\Delta \gamma}{\gamma_{0}} \sin \left(k_{s} l_{a}\right)
$$

can be imposed to the electrons exiting the modulator to account for the energy modulation. In the equation $l_{a}$ is the longitudinal electron coordinate at the exit of matching section, $\gamma_{0}$ is the reference/central energy of electron beam (scaled by electron's rest energy), $\Delta \gamma$ is the modulation depth, and $k_{s}$ is the wave number of the seed radiation.

For an electron with the initial coordinate vector defined by

$$
\vec{X}_{0}=\left(\begin{array}{c}
x_{0} \\
x_{0}^{\prime} \\
l_{0} \\
\delta_{0}
\end{array}\right)
$$

it is easy to show the electron's longitudinal coordinate at the exit of dispersion section can be written as

$$
\begin{aligned}
l_{b}= & l_{0}+C x_{0}+S x_{0}^{\prime}+D \delta_{0} \\
& +b_{56} \frac{\Delta \gamma}{\gamma_{0}} \sin \left[k_{s}\left(a_{51} x_{0}+a_{52} x_{0}^{\prime}+l_{0}+a_{56} \delta_{0}\right)\right]
\end{aligned}
$$

with

$$
\begin{aligned}
& C=b_{51} a_{11}+b_{51} m_{12} a_{21}+b_{52} a_{21}+a_{51} \\
& S=b_{51} a_{12}+b_{51} m_{12} a_{22}+b_{52} a_{22}+a_{52} \\
& D=b_{51} a_{16}+b_{51} m_{12} a_{26}+b_{52} a_{26}+a_{56}+b_{56} .
\end{aligned}
$$

To characterize the electron density modulation after the dispersion section, the bunching factor for the $h$ th harmonic is widely used, which is defined as [32]

$$
\begin{aligned}
b_{h} & =\left|\left\langle e^{-i h k_{s} l_{b}}\right\rangle\right| \\
& =\left|\int e^{-i h k_{s} l_{b}} F\left(x_{b}, x_{b}^{\prime}, l_{b}, \delta_{b}\right) d x_{b} d x_{b}^{\prime} d l_{b} d \delta_{b}\right| .
\end{aligned}
$$

Herein $F$ is the normalized electron distribution in the 4-dimensional phase space and the subscript " $b$ " denotes the coordinates at the exit of dispersion section.

Using the Liouville theorem which derives

$$
\begin{aligned}
& F\left(x_{b}, x_{b}^{\prime}, l_{b}, \delta_{b}\right) d x_{b} d x_{b}^{\prime} d l_{b} d \delta_{b} \\
& \quad=F\left(x_{0}, x_{0}^{\prime}, l_{0}, \delta_{0}\right) d x_{0} d x_{0}^{\prime} d l_{0} d \delta_{0},
\end{aligned}
$$

plugging in Eq. (6), and applying the Jacobi-Anger expansion [33], Eq. (9) becomes 


$$
\begin{aligned}
b_{h}= & \mid \int e^{-i h k_{s} l_{0}} e^{-i h k_{s} C x_{0}} e^{-i h k_{s} S x_{0}^{\prime}} e^{-i h k_{s} D \delta_{0}} \\
& \times \sum_{n=-\infty}^{\infty} J_{n}\left(-h k_{s} b_{56} \frac{\Delta \gamma}{\gamma_{0}}\right) e^{i n k_{s}\left(a_{51} x_{0}+a_{52} x_{0}^{\prime}+l_{0}+a_{56} \delta_{0}\right)} \\
& \times F\left(x_{0}, x_{0}^{\prime}, l_{0}, \delta_{0}\right) d x_{0} d x_{0}^{\prime} d l_{0} d \delta_{0} \mid
\end{aligned}
$$

Considering the case that all initial coordinates $\left(x_{0}, x_{0}^{\prime}\right.$, $l_{0}$, and $\delta_{0}$ ) are independent of each other and the bunch length is much longer than the modulation period, the integration over $l_{0}$ can be carried out directly. This yields nonvanishing bunching when $n=h$ with the bunching factor

$$
\begin{aligned}
b_{h}= & J_{h}\left(h k_{s}\left|b_{56}\right| \frac{\Delta \gamma}{\gamma_{0}}\right) \int e^{-i h k_{s} \bar{C} x_{0}} e^{-i h k_{s} \bar{S} x_{0}^{\prime}} \\
& \times e^{-i h k_{s} \bar{D} \delta_{0}} f\left(x_{0}, x_{0}^{\prime}, \delta_{0}\right) d x_{0} d x_{0}^{\prime} d \delta_{0},
\end{aligned}
$$

where $f$ is the normalized electron distribution in the 3 -dimensional projected space $\left(x, x^{\prime}, \delta\right)$ and

$$
\begin{aligned}
& \bar{C}=b_{51} a_{11}+b_{51} m_{12} a_{21}+b_{52} a_{21} \\
& \bar{S}=b_{51} a_{12}+b_{51} m_{12} a_{22}+b_{52} a_{22} \\
& \bar{D}=b_{51} a_{16}+b_{51} m_{12} a_{26}+b_{52} a_{26}+b_{56} .
\end{aligned}
$$

Assuming $x_{0}, x_{0}^{\prime}$, and $\delta_{0}$ have Gaussian distribution with the root mean square (RMS) values of $\sigma_{x}, \sigma_{x^{\prime}}$ and $\sigma_{\delta}$, respectively, Eq. (12) can then be integrated as

$b_{h}=J_{h}\left(h k_{s}\left|b_{56}\right| \frac{\Delta \gamma}{\gamma_{0}}\right) e^{-\frac{1}{2} h^{2} k_{s}^{2} \bar{C}^{2} \sigma_{x}^{2}} e^{-\frac{1}{2} h^{2} k_{s}^{2} \bar{S}^{2} \sigma_{x^{\prime}}^{2}} e^{-\frac{1}{2} h^{2} k_{s}^{2} \bar{D}^{2} \sigma_{\delta}^{2}}$.

To achieve a high bunching factor, the electron modulation needs to be optimized. However, the coefficients in Eq. (13) involves a lot of transfer matrix elements, which makes the situation rather complicated. This can be simplified by choosing $b_{51}=0$, in which case

$$
\begin{aligned}
\bar{C} & =b_{52} a_{21} \\
\bar{S} & =b_{52} a_{22} \\
\bar{D} & =b_{52} a_{26}+b_{56} .
\end{aligned}
$$

Among the three exponential factors in Eq. (14), the one representing the effect of electron energy spread has a maximum of unity when $\bar{D}=0$. Since $b_{56}$ should have a nonzero value otherwise $J_{h}\left(h k_{s}\left|b_{56}\right| \Delta \gamma / \gamma_{0}\right)=0$, nonvanishing matrix elements of $a_{26}$ and $b_{52}$ are needed, which indicates that dipole magnets should be included in the matching section to introduce angular dispersion $\left(a_{26}\right)$. This angular dispersion, together with a nonzero $b_{52}$, is vital for achieving high-efficient bunching when the amplitude of electron energy modulation is comparable to the initial uncorrelated energy spread, as will be discussed later.

The other two exponential factors can also be maximized to unity by choosing $\bar{C}=0$ and $\bar{S}=0$. This in turn requires $a_{21}=0$ and $a_{22}=0$, respectively, since $b_{52}$ has a nonzero value. It is more straightforward to make just one of them vanish in beam optics. Herein we choose $a_{21}=0$, which allows an injected electron beam with a larger horizontal size. Accordingly, the bunching factor reduces to

$$
b_{h}=J_{h}\left(h k_{s}\left|b_{56}\right| \frac{\Delta \gamma}{\gamma_{0}}\right) e^{-\frac{1}{2} h^{2} k_{s}^{2} a_{22} b_{52} \sigma_{x^{\prime}}^{2}}
$$

which has a similar form as presented in [24]. However, the result herein does not necessarily rely on a stringent assumption on the beam line. Consequently, it can be applied to more general cases, e.g., when the dipole magnets cannot be treated as thin lens. This is very important for the applications employing lower-energy electron beams.

\section{THE MODULATION SCHEME}

Based upon the above observations, we propose a scheme to generate strong subpicosecond density modulation in a low-energy (e.g., a few tens $\mathrm{MeV}$ ) electron beam, as illustrated in Fig. 1. For the matching section, one dipole magnet and two quadrupole magnets are used. The dipole magnet triggers the angular dispersion of the electron beam, while the two quadrupole magnets allow adjusting the strength of focusing $\left(a_{21}\right)$ and angular dispersion $\left(a_{26}\right)$. The energy modulator adopts a short undulator and a $\mathrm{kW}$-level (peak power) coherent $\mathrm{THz}$ radiation source. In this case, the electron beam gets slightly energy-modulated through interacting with the low-power $\mathrm{THz}$ seed radiation. The dispersion section is a dogleg comprising two bending magnets of opposite polarities and one quadrupole magnet in between. These magnets make $b_{51}$ vanish while providing the required $b_{56}$ to maximize the Bessel function in the bunching factor [see Eq. (14)]. After the dispersion section, the energy modulation is converted into density modulation. With proper set $a_{21}$ and $a_{26}, \bar{C}$ and $\bar{D}$ approach zero,

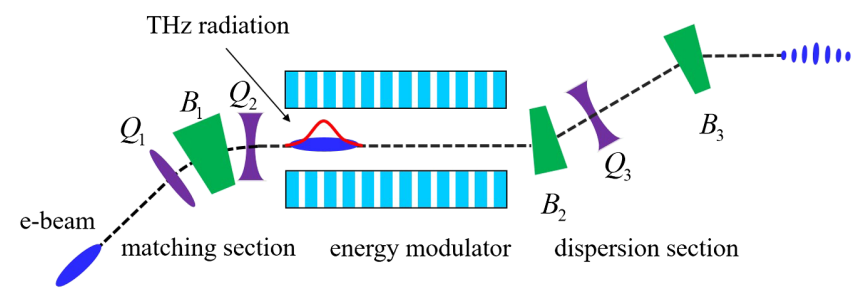

FIG. 1. An electron modulation scheme to generate strong subpicosecond density modulation in relativistic electron beams with low-power $\mathrm{THz}$ seed radiation. $B_{1}, B_{2}$, and $B_{3}$ are sector dipole magnets; $Q_{1}, Q_{2}$, and $Q_{3}$ are quadrupole magnets. 
respectively. Therefore, the effects of initial transverse size and uncorrelated energy spread on the modulation can be greatly suppressed.

The coherent $\mathrm{THz}$ radiation herein can be produced via stimulated polariton scattering in the polar nonlinear optical materials (such as $\mathrm{LiNbO}_{3}$ and $\mathrm{KTP}$ ), namely $\mathrm{THz}$ parametric amplifier (TPA) [25]. This technology has been well developed recently and can generate narrow-band $\mathrm{THz}$ radiation with the peak power above $1 \mathrm{~kW}$ when driven by a commercial infrared (e.g., $1064 \mathrm{~nm}$ ) 100s MW laser. Moreover, the $\mathrm{THz}$ radiation wavelength is tunable within 1-3 THz when using $\mathrm{LiNbO}_{3}$ crystal [26] and 3-6 THz when using KTP crystal [27].

As an example to demonstrate the scheme, we assume the input/injected electron beam has an energy of $30 \mathrm{MeV}$ and an energy spread of $0.1 \%$. We also assume the electron beam has a uniform current profile with a bunch length of $2.5 \mathrm{ps}$ and a bunch charge of $100 \mathrm{pC}$. For higher bunching factor, the electron beam is expected to have a low emittance and a small horizontal divergence, which are assumed to be $0.4 \mu \mathrm{m}$ (normalized) and $11 \mu \mathrm{rad}$, respectively. These parameters are realistic and readily available from many electron accelerators [34-36]. For the energy modulator, the planar undulator is supposed to have 20 periods with the period length of $4 \mathrm{~cm}$. The THz seed radiation is assumed to have a wavelength of $50 \mu \mathrm{m}(6 \mathrm{THz}$ in frequency), a peak power of $1 \mathrm{~kW}$, and a pulse duration of $10 \mathrm{ps}$. These parameters have been achieved in our TPA experiments. The THz seed is supposed to be a Gaussian beam, whose waist is $w_{0}=2.2 \mathrm{~mm}$ and located at the middle of undulator. It is worth noting that both the electron beam and $\mathrm{THz}$ seed radiation have relatively large transverse sizes, which would benefit their alignment and lead to a rather high tolerance to electron beam position jittering in the undulator. A summary of parameters for the electron beam and energy modulator is listed in Table I.

The parameters for the matching and dispersion sections are determined by solving the transfer matrix with a fast genetic algorithm. One set of results are listed in Table II. With the above parameters, the bunching factor at the fundamental wavelength is 0.422 according to Eq. (14). In the calculation, $\Delta \gamma / \gamma_{0}$ was evaluated using [37]

$$
\frac{\Delta \gamma}{\gamma_{0}}=\frac{a_{u}[J J]}{\gamma_{0}^{2}} \frac{L_{u}}{\kappa w_{0}} \sqrt{\frac{P_{s}[G W]}{4.35}}
$$

where $[J J]=J_{0}\left[\left(a_{u}^{2} / 2\right) /\left(1+a_{u}^{2}\right)\right]-J_{1}\left[\left(a_{u}^{2} / 2\right) /\left(1+a_{u}^{2}\right)\right]$, $L_{u}$ is the modulating undulator length, $P_{s}$ is the seed $\mathrm{THz}$ radiation power for energy modulation, and $\kappa$ is newly introduced herein to account for the radiation beam size variation in the undulator, which is defined as the ratio of the averaged size to the waist radius $w_{0}$. When the radiation beam waist is located at the middle of undulator, $\kappa=$ $\log \left(\zeta+\sqrt{\zeta^{2}+1}\right) / 2 \zeta+\sqrt{\zeta^{2}+1} / 2$ with $\zeta=L_{u} / 2 Z_{r}$.
TABLE I. Main electron beam and energy modulator parameters.

\begin{tabular}{lcc}
\hline \hline Parameter & Value & Unit \\
\hline Electron beam & 30 & $\mathrm{MeV}$ \\
Energy & 100 & $\mathrm{pC}$ \\
Bunch charge & 2.5 & $\mathrm{ps}$ \\
Bunch length & $0.1 \%$ & \\
Energy spread & 0.4 & $\mu \mathrm{m}$ \\
Normalized emittance $\epsilon_{n}$ & 11 & $\mu \mathrm{rad}$ \\
Horizontal divergence $\sigma_{x^{\prime}}$ & & \\
Seed radiation & 50 & $\mu \mathrm{m}$ \\
Wavelength $\lambda_{s}$ & 1 & $\mathrm{~kW}$ \\
Peak power $P_{s}$ & 10 & $\mathrm{ps}$ \\
Pulse length (FWHM) & 0.3 & $\mathrm{~m}$ \\
Rayleigh range $Z_{r}$ & 2.2 & $\mathrm{~mm}$ \\
Waist radius $w_{0}$ & & $\mathrm{~cm}$ \\
Undulator & 4 & \\
Period $\lambda_{u}$ & 20 & \\
Period number $N_{u}$ & 2.76 & \\
RMS deflecting parameter $a_{u}$ & & \\
\hline \hline
\end{tabular}

TABLE II. Main parameters of the beam optics in Fig. 1. Not labeled in the figure are drift sections $D_{1}$ between $Q_{1}$ and $B_{1}, D_{2}$ between $B_{1}$ and $Q_{2}, D_{3}$ between $B_{2}$ and $Q_{3}$, and $D_{4}$ between $Q_{3}$ and $B_{3}$.

\begin{tabular}{lcc}
\hline \hline Parameter & Value & Unit \\
\hline Matching section & & \\
Bending angle $\theta_{B_{1}}$ & 11 & degree \\
Bending length $L_{B_{1}}$ & 0.22 & $\mathrm{~m}$ \\
Quadrupole strength $K_{Q_{1}}$ & 2.00 & $\mathrm{~m}^{-2}$ \\
Quadrupole strength $K_{Q_{2}}$ & -4.09 & $\mathrm{~m}^{-2}$ \\
Quadrupole length $L_{Q 1, Q 2}$ & 0.1 & $\mathrm{~m}$ \\
Drift space length $L_{D_{1}, D_{2}}$ & 0.1 & $\mathrm{~m}$ \\
Dispersion section & & \\
Bending angle $\theta_{B_{2}, B_{3}}$ & 12 & degree \\
Bending length $L_{B_{2}, B_{3}}$ & 0.12 & $\mathrm{~m}$ \\
Quadrupole strength $K_{Q_{3}}$ & -6.67 & $\mathrm{~m}^{-2}$ \\
Quadrupole length $L_{Q_{3}}$ & 0.1 & $\mathrm{~m}$ \\
Drift space length $L_{D_{3}}$ & 0.33 & $\mathrm{~m}$ \\
Drift space length $L_{D_{4}}$ & 0.67 & $\mathrm{~m}$ \\
\hline \hline
\end{tabular}

In our example, the undulator length is $8 / 3$ times the radiation Rayleigh range $Z_{r}$, therefore $\kappa=1.245$.

\section{SIMULATION STUDY}

We use the code ELEGANT [38] to track the electron beam through the matching and dispersion sections with secondand third-order transport effects and incoherent synchrotron radiation (ISR) taken into account. The interaction between electron beam and $\mathrm{THz}$ radiation in the modulator is simulated using the time-dependent code GENESIS [39]. At the exit of the dispersion section, the electron coordinates are exported to calculate the bunching factor according to Eq. (8), which is referred to as the simulated 


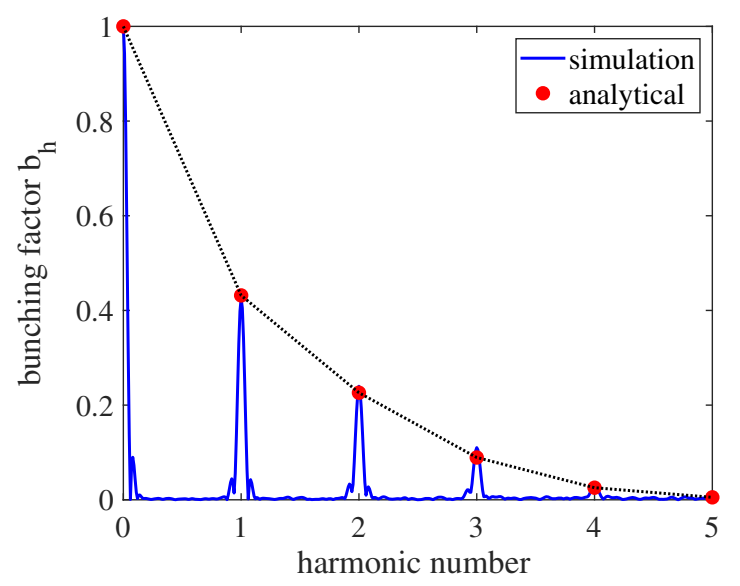

FIG. 2. Comparison of the bunching factors obtained via simulation and analytical formula.

bunching factor hereafter. Figure 2 shows a comparison of the simulated bunching factors to the analytical result [calculated using Eq. (14)] for various harmonic numbers. From the figure, one can see good agreement between the simulation and analytical formula. At the fundamental wavelength, the simulated bunching factor is 0.424 , which is very close to the analytical result.

So far both analytical method and simulation have shown that strong sub-picosecond electron bunching can be generated by modulating the electron beam via a $\mathrm{kW}$ (peak power) $\mathrm{THz}$ radiation. To illustrate the physical picture of this strong bunching, we plot the longitudinal phase space distribution of the electron beam at the exits of energy modulator and dispersion section in Fig. 3. For clarity, we slice the electrons according to their angular divergence $x^{\prime}$ at the entrance of the energy modulator in Fig. 3(a) and 3(d). Herein $x^{\prime}$ can represent the intrinsic energy deviation of electrons, since the matching section induced angular dispersion is much larger than the initial divergence. This is clearly shown in Fig. 3(a) and 3(d), where all electron slices become distinct "energy layers" in the longitudinal phase space.

From Fig 3(a), one can see obvious energy modulation in each layer. According to Eq. (17), the energy modulation amplitude is $1.6 \times 10^{-4}$, which is only $1 / 6$ of the beam's uncorrelated energy spread. In typical electron density modulation cases (e.g., High-Gain Harmonic Generation), such an energy modulation is incapable of evolving into efficient density modulation. With this scheme, however, the effect of initial energy deviation on electrons' path length will be cancelled out since $\bar{D}=0$. Therefore, in ideal case, only the modulation induced energy deviation contributes to the bunching process and strong density modulation is eventually formed after the dispersion section. This is clearly shown in Fig. 3(d), where the energy modulation of each layer is converted into density modulation, while the process is nearly independent of the layers' energy.

As mentioned above, to generate high density modulation via this scheme, the electron beam is expected to have a low emittance and a small horizontal divergence. To investigate the impact of emittance and horizontal divergence on the bunching factor, we carried out three sets of simulation with the normalized emittance of $0.4,0.6$, and $0.8 \mu \mathrm{m}$, respectively. For each set of simulation, the initial horizontal angular divergence $\sigma_{x}^{\prime}$ is scanned from 3 to $30 \mu \mathrm{rad}$, and accordingly the horizontal beam size is varied to maintain a same emittance. All other parameters, including those of the matching section, energy modulator, and dispersion section, are kept unchanged. The simulation results are plotted in Fig. 4. We can see from the figure that for all cases the bunching factor reaches its maximum when $\sigma_{x^{\prime}}$ is around $11 \mu \mathrm{rad}$. This is a balance between the horizontal divergence and beam size - as the divergence decreases, the beam size would increase accordingly, which

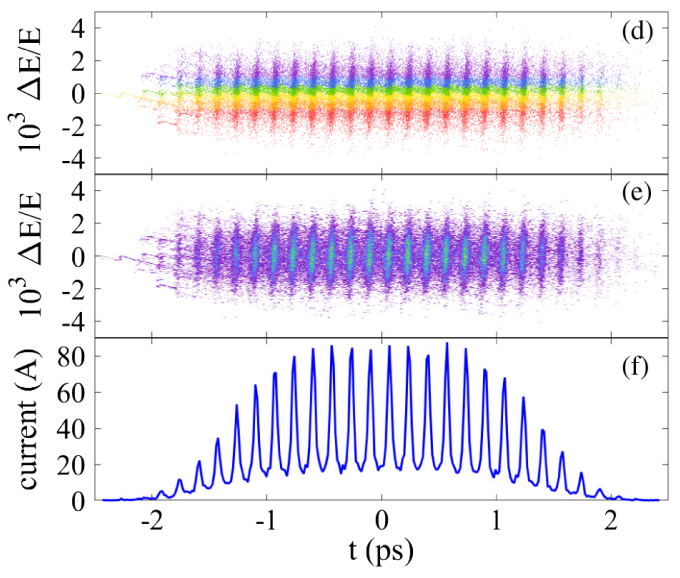

FIG. 3. Longitudinal phase space distribution of the electron beam at the exit of energy modulator (a-c) and dispersion section (d-f). In (a) and (d), the electrons are grouped into 6 "layers" according to their angular divergence $x^{\prime}$ at the entrance of energy modulator, and electrons in each layer are represented in a different color. (b) and (d) are pseudocolor plots of electron density distribution, while (c) and (f) are current profiles. 


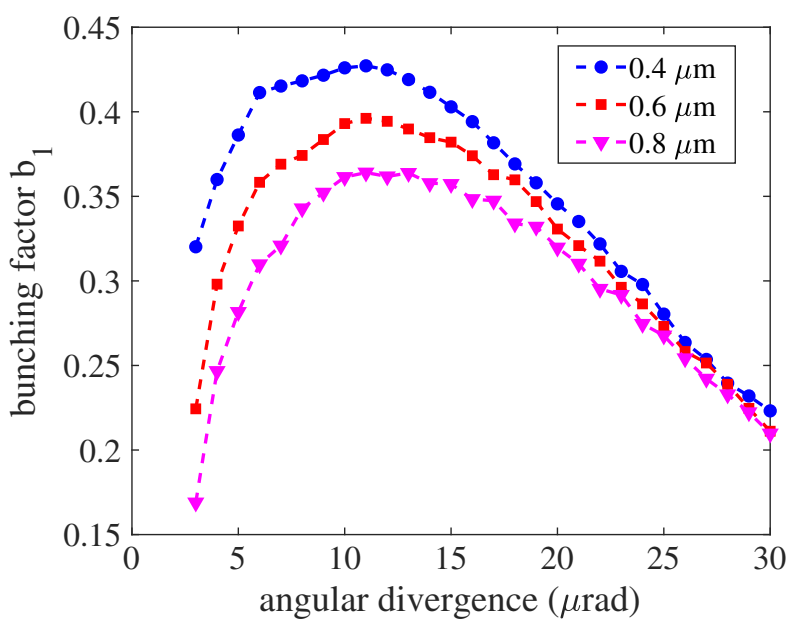

FIG. 4. Bunching factor at fundamental wavelength for various horizontal divergence and emittance.

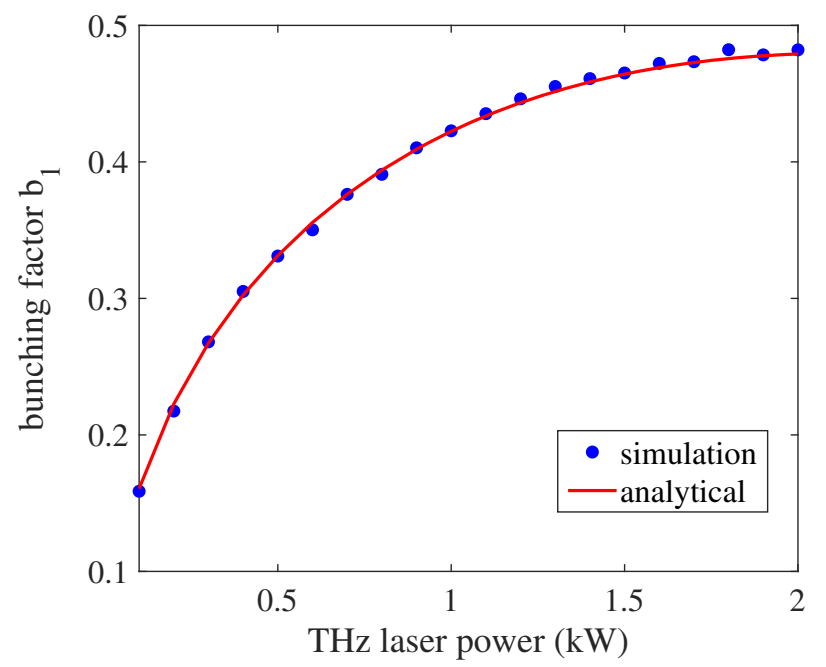

FIG. 5. Bunching factor at fundamental wavelength as a function of $\mathrm{THz}$ seed radiation power.

may reduce the electron-radiation coupling in the energy modulator. Nevertheless, the bunching factor remains relatively stable when $\sigma_{x^{\prime}}$ changes within a range $\sim 10 \mu \mathrm{rad}$. The figure also shows that when the emittance grows to $0.8 \mu \mathrm{m}$, the bunching factor can still reach 0.36 .

We have also investigated the impact of seed radiation power on the bunching factor. Figure 5 shows the simulation results, where all other parameters are kept unchanged. This figure indicates that a considerable bunching factor can be achieved even if the seed radiation power is reduced to $100 \mathrm{~s}$ $\mathrm{W}$ and the dispersion strength does not match the energy modulation amplitude. From the figure we can also infer that the bunching factor is rather stable against seed radiation power fluctuation at $10 \%$ level-for TPA, the expected fluctuation is within $2 \%$.

Finally, we briefly investigate the wavelength tunability of this scheme while keeping the transport optics (including

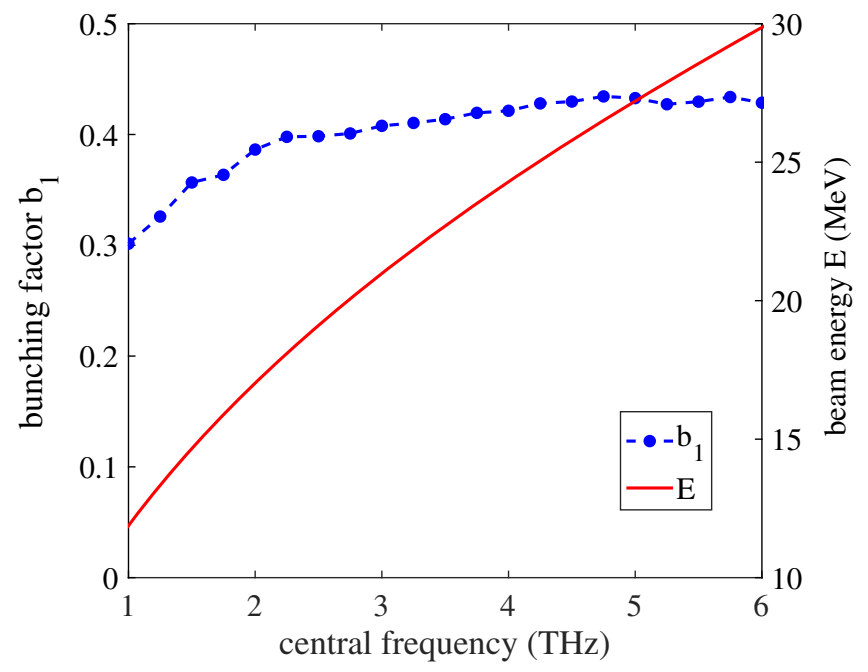

FIG. 6. Bunching factor for different frequency with the same beam line.

the matching and dispersion sections) unchanged. In the simulation, the modulation wavelength is scanned from 1 to $6 \mathrm{THz}$ with a step size of $0.25 \mathrm{THz}$, the electron energy is varied accordingly to maintain a same $a_{u}$ of the energy modulator, and the electron beam Twiss parameters and the $\mathrm{THz}$ seed radiation waist radius are adjusted to adapt these changes. Figure 6 presents the simulation result. As shown in the figure, a bunching factor at the level of 0.4 can be obtained over the frequency range from 1 to $6 \mathrm{THz}$.

\section{THZ SUPERRADIANT UNDULATOR RADIATION}

Now let us consider the superradiant undulator radiation of the bunched electron beam. The fundamental radiation power for such a beam traversing a undulator distance $L_{u}$ can be calculated using [40]

$$
P=\frac{b_{1}^{2}}{4} I_{p k}^{2} L_{u}^{2}\left(\frac{a_{u}}{\gamma_{0}}\right)^{2} \frac{Z_{0}}{\mathcal{A}},
$$

where $Z_{0}=377 \Omega$ is the vacuum impedance, $I_{p k}$ is the peak current of electron beam, and $\mathcal{A}$ is the effective mode area. It should be noted that Eq. (18) applies to spontaneous radiation only. Here we take the $6 \mathrm{THz}$ case as an example. We assume a 20-period undulator with a period length of $5 \mathrm{~cm}$ and a deflecting parameter of 2.47 which is resonant at $6 \mathrm{THz}$. The effective mode area can be estimated according to the fundamental Gaussian mode waist at the undulator center (assuming $L_{u}$ is twice the Rayleigh range), i.e., $\mathcal{A}=\pi w_{0}^{2} / 2=\lambda L_{u} / 4$. The output THz radiation power is therefore evaluated to be $15 \mathrm{MW}$, which is $1.5 \times 10^{4}$ times the seed radiation power. The radiation is expected to have a full spectral width of $5 \%$, depending on the undulator period number [32]. At last we want to point out that the chromatic transport in this scheme would lead 
to an increase of electron beam emittance. However, this issue will not have any significant impact on $\mathrm{THz}$ undulator radiation whose wavelength is much larger than the geometric emittance of electron beam [40].

\section{SUMMARY}

In this paper, we have proposed a scheme for generating strong electron density modulation at $\mathrm{THz}$ frequency using an external $\mathrm{THz}$ source directly. We have also developed a complete analytical model and presented an example of design according to the model for this scheme. Analytical calculation at $6 \mathrm{THz}$ (modulation frequency) shows that a bunching factor above 0.4 can be reached. Numerical study has been carried out to validate the results and investigate the impact of some sensitive parameters. The simulations agree well with the analytical model and show that the proposed scheme can achieve a bunching factor above 0.35 even as the electron beam emittance doubles or the seed radiation power reduces by $40 \%$. The wavelength tunability has also been investigated through simulation, which indicates that a bunching factor at the level of 0.4 can be obtained over the frequency range from 1 to $6 \mathrm{THz}$.

\section{ACKNOWLEDGMENTS}

We thank Chao Feng for very helpful discussions. This work is supported by National Key Research and Development Program of China (Grants No. 2017YFA0701000 and No. 2016YFA0401904), National Natural Science Foundation of China (Grant No. 11735002), and High-performance Computing Platform of Peking University.

[1] M. Tonouchi, Cutting-edge terahertz technology, Nat. Photonics 1, 97 (2007).

[2] B. Ferguson and X.-C. Zhang, Materials for terahertz science and technology, Nat. Mater. 1, 26 (2002).

[3] H. A. Hafez, X. Chai, A. Ibrahim, S. Mondal, D. Férachou, $\mathrm{X}$. Ropagnol, and T. Ozaki, Intense terahertz radiation and their applications, J. Opt. (Paris) 18, 093004 (2016).

[4] G. L. Carr, M. C. Martin, W. R. McKinney, K. Jordan, G. R. Neil, and G. P. Williams, High-power terahertz radiation from relativistic electrons, Nature (London) 420, 153 (2002).

[5] Z. Wu, A.S. Fisher, J. Goodfellow, M. Fuchs, D. Daranciang, M. Hogan, H. Loos, and A. Lindenberg, Intense terahertz pulses from SLAC electron beams using coherent transition radiation, Rev. Sci. Instrum. 84, 022701 (2013).

[6] B. Green, S. Kovalev, V. Asgekar, G. Geloni, U. Lehnert, T. Golz, M. Kuntzsch, C. Bauer, J. Hauser, J. Voigtlaender et al., High-field high-repetition-rate sources for the coherent THz control of matter, Sci. Rep. 6, 22256 (2016).

[7] W. Li, Z. He, Y. Lu, R. Huang, W. Liu, Q. Jia, and L. Wang, Tunable $\mathrm{THz}$ radiation source from dielectric loaded waveguide excited by nonrelativistic electron bunch trains, Phys. Rev. Accel. Beams 19, 104701 (2016).

[8] A. Cianchi, D. Alesini, M. P. Anania, A. Bacci, M. Bellaveglia, M. Castellano, E. Chiadroni, D. Di Giovenale, G. P. Di Pirro, M. Ferrario et al., Six-dimensional measurements of trains of high brightness electron bunches, Phys. Rev. ST Accel. Beams 18, 082804 (2015).

[9] Z. Zhang, L. Yan, Y. Du, W. Huang, C. Tang, and Z. Huang, Generation of high-power, tunable terahertz radiation from laser interaction with a relativistic electron beam, Phys. Rev. Accel. Beams 20, 050701 (2017).

[10] Z. Zhang, L. Yan, Y. Du, Z. Zhou, X. Su, L. Zheng, D. Wang, Q. Tian, W. Wang, J. Shi et al., Tunable HighIntensity Electron Bunch Train Production Based on Nonlinear Longitudinal Space Charge Oscillation, Phys. Rev. Lett. 116, 184801 (2016).

[11] Y. Lurie, A. Friedman, and Y. Pinhasi, Single pass, THz spectral range free-electron laser driven by a photocathode hybrid rf linear accelerator, Phys. Rev. ST Accel. Beams 18, 070701 (2015).

[12] P. Muggli, V. Yakimenko, M. Babzien, E. Kallos, and K. P. Kusche, Generation of Trains of Electron Microbunches with Adjustable Subpicosecond Spacing, Phys. Rev. Lett. 101, 054801 (2008).

[13] Y.-E. Sun, P. Piot, A. Johnson, A. H. Lumpkin, T. J. Maxwell, J. Ruan, and R. Thurman-Keup, Tunable Subpicosecond Electron-Bunch-Train Generation Using a Transverse-To-Longitudinal Phase-Space Exchange Technique, Phys. Rev. Lett. 105, 234801 (2010).

[14] Y.-C. Huang, Desktop megawatt superradiant free-electron laser at terahertz frequencies, Appl. Phys. Lett. 96, 231503 (2010).

[15] J. M. Byrd, Z. Hao, M. C. Martin, D. S. Robin, F. Sannibale, R. W. Schoenlein, A. A. Zholents, and M. S. Zolotorev, Tailored Terahertz Pulses from a Laser-Modulated Electron Beam, Phys. Rev. Lett. 96, 164801 (2006).

[16] S. Bielawski, C. Evain, T. Hara, M. Hosaka, M. Katoh, S. Kimura, A. Mochihashi, M. Shimada, C. Szwaj, T. Takahashi et al., Tunable narrowband terahertz emission from mastered laser-electron beam interaction, Nat. Phys. 4, 390 (2008).

[17] S. Antipov, C. Jing, M. Fedurin, W. Gai, A. Kanareykin, K. Kusche, P. Schoessow, V. Yakimenko, and A. Zholents, Experimental Observation of Energy Modulation in Electron Beams Passing through Terahertz Dielectric Wakefield Structures, Phys. Rev. Lett. 108, 144801 (2012).

[18] S. Antipov, M. Babzien, C. Jing, M. Fedurin, W. Gai, A. Kanareykin, K. Kusche, V. Yakimenko, and A. Zholents, Subpicosecond Bunch Train Production for a Tunable mJ Level THz Source, Phys. Rev. Lett. 111, 134802 (2013).

[19] L. Zhao, T. Jiang, C. Lu, R. Wang, Z. Wang, P. Zhu, Y. Shi, W. Song, X. Zhu, C. Jing et al., Few-femtosecond electron beam with terahertz-frequency wakefield-driven compression, Phys. Rev. Accel. Beams 21, 082801 (2018).

[20] D. Xiang and G. Stupakov, Enhanced tunable narrow-band $\mathrm{THz}$ emission from laser-modulated electron beams, Phys. Rev. ST Accel. Beams 12, 080701 (2009).

[21] M. Dunning, C. Hast, E. Hemsing, K. Jobe, D. McCormick, J. Nelson, T. O. Raubenheimer, K. Soong, Z. Szalata, D. Walz et al., Generating Periodic Terahertz Structures in a Relativ- 
istic Electron Beam through Frequency Down-Conversion of Optical Lasers, Phys. Rev. Lett. 109, 074801 (2012).

[22] S. Kumar, D.-E. Kim, and H.-S. Kang, Tunable THz radiation generation using density modulation of a relativistic electron beam, Nucl. Instrum. Methods Phys. Res., Sect. A 729, 19 (2013).

[23] Z. Wang, D. Huang, Q. Gu, Z. Zhao, and D. Xiang, Echoenabled tunable terahertz radiation generation with a lasermodulated relativistic electron beam, Phys. Rev. ST Accel. Beams 17, 090701 (2014).

[24] C. Feng and Z. Zhao, A storage ring based free-electron laser for generating ultrashort coherent EUV and x-ray radiation, Sci. Rep. 7, 4724 (2017).

[25] K. Kawase, J. ichi Shikata, and H. Ito, Terahertz wave parametric source, J. Phys. D 35, R1 (2002).

[26] Y. Takida, J.-i. Shikata, K. Nawata, Y. Tokizane, Z. Han, M. Koyama, T. Notake, S. Hayashi, and H. Minamide, Terahertz-wave parametric gain of stimulated polariton scattering, Phys. Rev. A 93, 043836 (2016).

[27] W. Wang, Z. Cong, X. Chen, X. Zhang, Z. Qin, G. Tang, N. Li, C. Wang, and Q. Lu, Terahertz parametric oscillator based on $\mathrm{KTiOPO}_{4}$ crystal, Opt. Lett. 39, 3706 (2014).

[28] H. Hamster, A. Sullivan, S. Gordon, and R. W. Falcone, Short-pulse terahertz radiation from high-intensity-laserproduced plasmas, Phys. Rev. E 49, 671 (1994).

[29] B. Liu, H. Bromberger, A. Cartella, T. Gebert, M. Först, and A. Cavalleri, Generation of narrowband, high-intensity, carrier-envelope phase-stable pulses tunable between 4 and 18 THz, Opt. Lett. 42, 129 (2017).

[30] K. L. Brown, A First and Second Order Matrix Theory for the Design of Beam Transport Systems and Charged Particle Spectrometers, Adv. Part. Phys. 1, 71 (1982).

[31] V. Balandin and N. Golubeva, Approximate matrices for modeling the focusing of the undulator periods and undulator end fields, in Proceedings of IPAC17,
Copenhagen, Denmark, International Particle Accelerator Conference (JACoW, Geneva, Switzerland, 2017), pp. 686-688, https://doi.org/10.18429/JACoW-IPAC2017MOPIK069.

[32] K.-J. Kim, Z. Huang, and R. Lindberg, Synchrotron Radiation and Free-Electron Lasers: Principles of Coherent X-Ray Generation (Cambridge University Press, Cambridge, England, 2017).

[33] A. Cuyt, V. Petersen, B. Verdonk, H. Waadeland, and W. Jones, Handbook of Continued Fractions for Special Functions (Springer, New York, 2008).

[34] Y. Ding, A. Brachmann, F.-J. Decker, D. Dowell, P. Emma, J. Frisch, S. Gilevich, G. Hays, P. Hering, Z. Huang et al., Measurements and Simulations of Ultralow Emittance and Ultrashort Electron Beams in the Linac Coherent Light Source, Phys. Rev. Lett. 102, 254801 (2009).

[35] C. Gulliford, A. Bartnik, I. Bazarov, L. Cultrera, J. Dobbins, B. Dunham, F. Gonzalez, S. Karkare, H. Lee, H. Li et al., Demonstration of low emittance in the Cornell energy recovery linac injector prototype, Phys. Rev. ST Accel. Beams 16, 073401 (2013).

[36] S. Bettoni, M. Pedrozzi, and S. Reiche, Low emittance injector design for free electron lasers, Phys. Rev. ST Accel. Beams 18, 123403 (2015).

[37] E. Hemsing, G. Stupakov, D. Xiang, and A. Zholents, Beam by design: Laser manipulation of electrons in modern accelerators, Rev. Mod. Phys. 86, 897 (2014).

[38] M. Borland, Simple method for particle tracking with coherent synchrotron radiation, Phys. Rev. ST Accel. Beams 4, 070701 (2001).

[39] S. Reiche, GENESIS 1.3: a fully 3D time-dependent FEL simulation code, Nucl. Instrum. Methods Phys. Res., Sect. A 429, 243 (1999).

[40] A. Gover, Superradiant and stimulated-superradiant emission in prebunched electron-beam radiators. I. Formulation, Phys. Rev. ST Accel. Beams 8, 030701 (2005). 